\title{
Time-resolved X-ray Absorption Spectroscopy of Photoinduced Insulator-Metal Transition in a Colossal Magnetoresistive Manganite
}

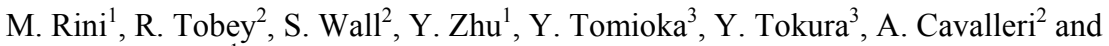 \\ R.W. Schoenlein ${ }^{1}$ \\ ${ }^{1}$ Lawrence Berkeley National Laboratory, Berkeley, CA 94720, USA \\ ${ }^{2}$ Department of Physics, Clarendon Laboratory, University of Oxford, Oxford OX1 3PU, \\ United Kingdom \\ ${ }^{3}$ Correlated Electron Research Center, AIST, Tsukuba, Ibaraki, 305-8562 Japan \\ E-mail: mrini@lbl.gov
}

\begin{abstract}
We studied the ultrafast insulator-metal transition in a manganite by means of picosecond X-ray absorption at the $\mathrm{O} \mathrm{K}$ - and $\mathrm{Mn} \mathrm{L}$-edges, probing photoinduced changes in $\mathrm{O}$ $2 p$ and Mn-3d electronic states near the Fermi level.
\end{abstract}

Manganites pose an important testing ground for studying electron correlation phenomena and exhibit close analogies with a wide variety of materials in the correlated-electron family, including high- $\mathrm{T}_{\mathrm{c}}$ superconducting cuprates. In these systems, the strong interplay between charge, spin, orbital and lattice degrees of freedom results in rich phase diagrams. Phase competition at the boundaries between these phases leads to a number of remarkable phenomena including colossal magnetoresistance (CMR) [1]. Arguably, the most striking aspect of the physics of manganites is the occurrence of a number of metal-insulator transitions, initiated for instance via perturbations of temperature, magnetic field, pressure, and irradiation with light [1].

The importance of ultrafast time-resolved measurements in understanding complex materials lies in the capability to excite them (perturbatively) on time-scales shorter than the underlying correlations and then disentangle the interactions by probing their time response as the correlation develops. Ultrafast x-ray techniques in particular present an exceptional opportunity for studying the correlated interplay between atomic and electronic structures.

Here we applied time-resolved x-ray absorption spectroscopy to study the photoinduced phase-transitions in the CMR $\operatorname{Pr}_{1-\mathrm{x}} \mathrm{Ca}_{\mathrm{x}} \mathrm{MnO}_{3}$ (Fig. 1). In $\operatorname{Pr}_{1-\mathrm{x}} \mathrm{Ca}_{\mathrm{x}} \mathrm{MnO}_{3}$, at the optimal doping level $\mathrm{x}=0.3$, the insulating phase adjoins a "hidden" metallic state of the system characterized by enormous changes in resistivity [2]. The metallic phase can be reached by application of external perturbations such as a magnetic field, as in the CMR effect shown in Fig. 1(b). An important development has been the demonstration that a first-order insulator-metal phase transition can be induced optically, by the photoinjection of carriers into the insulating state [3]. Even more remarkably, our recent time-resolved reflectivity and transport studies (Fig. 1 (c)) show that the transition to the metallic state occurs on a sub-ps time scale and can be initiated by selective vibrational excitation of the Mn-O stretching mode [4]. 

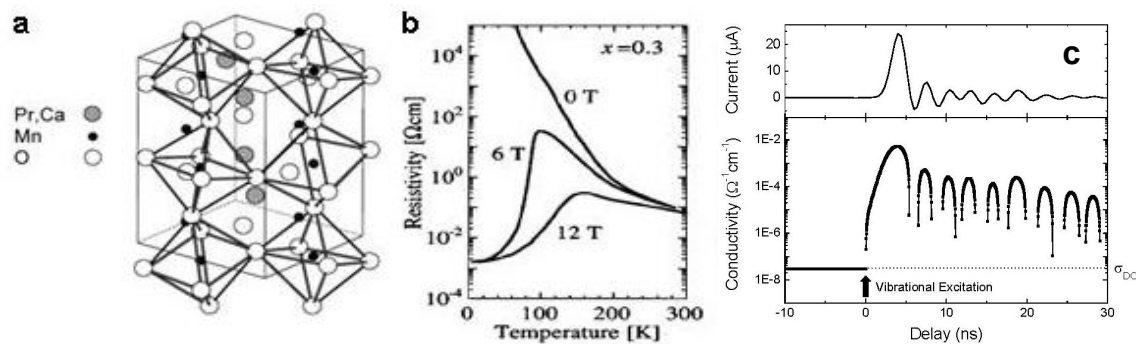

Fig. 1. (a) Perovskite crystal structure of $\operatorname{Pr}_{0.7} \mathrm{Ca}_{0.3} \mathrm{MnO}_{3}$. (b) $\mathrm{CMR}$ effect in $\mathrm{Pr}_{0.7} \mathrm{Ca}_{0.3} \mathrm{MnO}_{3}$ shown by temperature dependent resistivity measurements under $0,6,12 \mathrm{~T}$ magnetic field [2]. (c) 5-order-of-magnitude changes in sample resistivity are observed when the Mn-O stretching phonon mode is resonantly excited by $17-\mu \mathrm{m}$ laser pulses. X-ray measurements will give better insights into the dynamics of relevant electronic states and of changes in local structure distorsion following vibrational excitation.

We studied the photoinduced phase transition in $\mathrm{Pr}_{0.7} \mathrm{Ca}_{0.3} \mathrm{MnO}_{3}$ by means of infrared-pump/X-ray absorption spectroscopy (time-resolved XANES). X-ray absorption spectroscopy has proven to be a powerful tool for addressing important questions about the physics of manganites, providing important insights into the electronic structure and the lattice distortions of these oxides [5]. Since the valence and conduction bands in CMR manganites are comprised of hybridized Mn-3d and $\mathrm{O}-2 \mathrm{p}$ states, complementary information can be obtained from time-resolved XANES measurements at the $\mathrm{O} \mathrm{K}$-edge and $\mathrm{Mn} \mathrm{L}$-edges. O K-edge spectra probe unoccupied states of mixed O-2p and Mn-3d character near the Fermi level, and the low-energy pre-edge structure directly reflects changes in the hybridization of these states [5], which influences charge localization/conductivity and d-electron hopping probability. Recent static measurements in manganites have shown that the O K-edge XANES spectrum exhibits substantial changes across the insulator-metal transition and is particularly sensitive to the presence of local Jahn-Teller distorsions [5]. On the other hand, Mn L-edge XANES probes the unoccupied states of predominantly metal-3d character and the chemical edge shift provides information about changes in the $\mathrm{Mn}$ oxidation state [6]. Time-resolved XANES measurements are thus particularly sensitive to local structural distortion of the Mn-O complex resulting from the photoexcitation, e.g. polaron effects or changes in the Mn-O-Mn bond angle which strongly influence the electron hopping probability [1]. Local distortions will be manifest in changes to the $3 d-2 p$ hybridization which is at the heart of the insulatormetal transition in these compounds.

$\mathrm{Pr}_{0.7} \mathrm{Ca}_{0.3} \mathrm{MnO}_{3}$ samples were excited with $100 \mathrm{fs}$ pulses at $1.55 \mathrm{eV}$. We followed the phase transition dynamics by probing with $70 \mathrm{ps}$ X-ray pulses tuned through the $\mathrm{O}$ K-edge at $530 \mathrm{eV}$ and the Mn L-edges at $640 \mathrm{eV}$. Measurements were taken in Total Electron Yield (TEY) mode at $80 \mathrm{~K}$. Following 800-nm excitation, we observed photoinduced absorption changes at the O K-edge and at the Mn L-edge (Fig 2(a,b)). Fig. 2(c) shows time-dependent pump-probe signals at probe energy of $529 \mathrm{eV}$, measured as a function of the delay time between laser and X-ray pulses. The solid 
line is a fit of the data using a cross correlation width of $70 \mathrm{ps,} \mathrm{corresponding} \mathrm{to} \mathrm{the}$ temporal resolution dictated by the X-ray pulse width. The intensity dependence of the measured signals exhibits the same features as in previous femtosecond reflectivity studies [4], with a threshold and saturation behavior which is characteristic of a phase transformation to the metallic state. The extension of these experiments to the femtosecond regime techniques is in progress and will monitor the phase-transition dynamics with appropriate time-resolution and access time scales faster than electron-lattice relaxation processes, disentangling effects due to the phase transition from laser-induced heating effects.
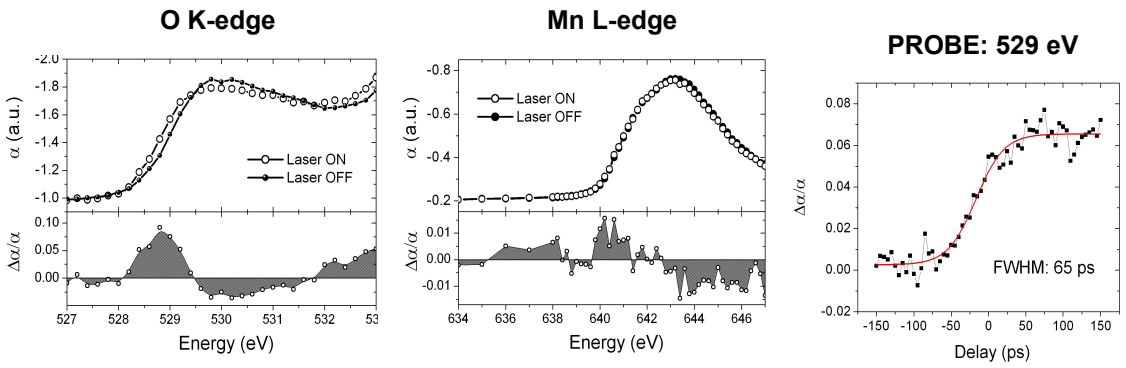

Fig. 2. Picosecond time-resolved XANES in $\mathrm{Pr}_{0.7} \mathrm{Ca}_{0.3} \mathrm{MnO}_{3}$. (a,b) Upper panels: Static absorption spectra (solid circles) at the O K-edge (a) and Mn L-edge (b) and spectra measured $300 \mathrm{ps}$ after $800-\mathrm{nm}$ excitation (open circles) at a fluence of $\sim 30 \mathrm{~mJ} / \mathrm{cm}^{2}$. Lower panels: corresponding relative change of absorption $(\Delta \alpha / \alpha)$. (c) Relative change of absorption $(\Delta \alpha / \alpha)$ as a function of delay between the $800-\mathrm{nm}$ pump and the X-ray probe pulses, measured at the representative wavelength of $529 \mathrm{eV}$. Measurements were taken at $80 \mathrm{~K}$ in Total Electron Yield (TEY) mode.

Acknowledgements. This work was supported by the U.S. Department of Energy under Contract No. DE-AC02-05CH11231

1 Y. Tokura, Colossal magnetoresistive oxides (Gordon and Breach Science Publishers, 2000).

2 Y. Tomioka et al., Phys. Rev. B 53, 1689-1692 (1996).

3 M. Fiebig et al., Science 280, 1925-1928 (1998).

4 M. Rini et al., Nature, 449, 72-72 (2007).

5 N. Mannella et al., Phys. Rev. B 71, 125117 (2005).

6 Y. S. Lee et al., Phys. Stat. Sol. A, 196, 70-73 (2003). 